\title{
Commentaire général et comparatif de Racine (1818): préface et articles 10-17
}

\section{Pierre Fontanier}

\section{(2) OpenEdition}

1 Journals

Édition électronique

URL : http://journals.openedition.org/rhetorique/158

DOI : 10.4000/rhetorique. 158

ISSN : 2270-6909

Éditeur

UGA Éditions/Université Grenoble Alpes

Édition imprimée

ISBN : 978-2-84310-255-4

\section{Référence électronique}

Pierre Fontanier, « Commentaire général et comparatif de Racine (1818) : préface et articles 10-17 », Exercices de rhétorique [En ligne], 1 | 2013, mis en ligne le 18 novembre 2013, consulté le 12 septembre 2020. URL : http://journals.openedition.org/rhetorique/158; DOI : https://doi.org/10.4000/rhetorique. 158

Ce document a été généré automatiquement le 12 septembre 2020.

\section{(c) (†) (ㅇ)}

Les contenus de la revue Exercices de rhétorique sont mis à disposition selon les termes de la Licence Creative Commons Attribution - Pas d'Utilisation Commerciale - Partage dans les Mêmes Conditions 4.0 International. 


\title{
Commentaire général et comparatif de Racine (1818) : préface et articles 10-17
}

\author{
Pierre Fontanier
}

\section{Préface du Commentaire [extraits]}

1 La Grammaire la plus étendue et la plus complète ne peut guère offrir qu'un enseignement théorique; elle ne peut donner sur presque tous les points que des principes généraux et spéculatifs: et il y a souvent si loin de ces principes à l'application! ou ces principes sont continuellement susceptibles de tant d'exceptions ou de modifications diverses, suivant les circonstances !...

Comment donc suppléer à l'insuffisance des Grammaires? Le meilleur moyen, assurément, et même l'unique peut-être, c'est la lecture assidue et réfléchie des Classiques, non des Classiques bornés à leur texte pur et simple, mais accompagnés d'observations ou remarques où se trouve particulièrement relevé tout ce que la diction et le style peuvent avoir de plus répréhensible : car quel est l'auteur si parfait à qui on ne puisse reprocher ni défauts ni fautes ? [...] Or, quel est celui de tous qui, pour la diction, pour le style, peut le disputer à Racine, ou même soutenir la comparaison avec lui ? Racine n'est-il pas, sous ce rapport, éminemment Classique, le Classique par excellence ? N'est-il pas généralement regardé, pour la perfection en ce genre, comme le Virgile français ? [...] Et y en a-t-il un sur qui l'on ait autant médité, autant écrit, sur qui l'on ait fait autant de volumes, et qui se présente environné d'un aussi nombreux cortège de Commentateurs? [...]

Ne serait-il donc pas à propos qu'il y eût sur Racine, sur cet écrivain supérieur, incomparable, le premier de nos Classiques, et notre plus grand maitre en fait de langue, comme en fait de poésie, un ouvrage qui présentât recueillies, rapprochées, et réunies en un seul corps, toutes les observations ou remarques les plus importantes qu'on a pu faire jusqu'ici sur sa diction et sur son style ? Cet ouvrage n'aurait-il pas une 
utilité encore plus marquée et plus complète, si, à ces remarques ou observations en étaient jointes, au besoin, d'autres destinées à les justifier, à les éclaircir, à les rectifier, ou à les contredire ? [...]

4 Cet ouvrage si utile, si nécessaire, dont je viens de tracer la première idée, j'ai osé l'entreprendre, et c'est celui que j'offre ici au Public. Il est loin, sans doute, d'avoir reçu de ma main toute la perfection qu'eût pu lui donner un de ces maîtres en littérature connus par la délicatesse de leur goût et l'élégance de leur plume. Mais, à moins que je n'aie travaillé tout à fait en dépit du bon sens, il ne peut pas être sans quelque mérite, et même sans un mérite assez marqué. En effet, n'a-t-il pas dans tous ces extraits des divers Commentaires dont il se compose en grande partie, un fonds essentiellement bon, et dont la réputation était déjà faite d'avance ? [...] Ce que j'y ai mis de mon propre fonds pourra sans doute ne pas [...] paraitre toujours excellent ; mais, en produisant sur chaque question toutes les raisons pour et contre, j'aurai fourni les moyens, non seulement de juger qui a tort de moi ou de ceux que j'ose quelquefois me permettre de contredire, mais même de reconnaître et de relever à l'instant mes méprises ou mes erreurs.

5 Par toutes ces considérations, et d'après le but que je me suis proposé d'offrir ce Commentaire comme une sorte de supplément aux Grammaires, et comme une espèce de Cours pratique, bien que sans doute raisonné et philosophique, de notre langue, je n'ai pas cru devoir y joindre le texte entier du poète, c'est-à-dire, tout son théâtre. Qui est-ce qui n'a pas un Racine ? et quels sont ceux qui, en ayant déjà un, peuvent tant se soucier d'en acquérir un nouveau? D'ailleurs, le texte, joint au Commentaire, eût pris une partie de l'attention que celui-ci demande, l'eût rendu un objet purement secondaire, eût fait perdre de vue nombre de remarques, et, en séparant, en isolant les unes des autres, celles qui vont ensemble, eût souvent empêché de les voir à la fois, d'en saisir les rapports, d'en apprécier avec justesse la valeur, le mérite. Et enfin ce n'est pas précisément un Commentaire sur Racine que j'ai voulu faire, mais j'ai voulu faire d'un Commentaire sur Racine un ouvrage distinct et séparé, non moins particulièrement destiné à servir pour l'étude de la langue, qu'un Dictionnaire, une Grammaire, un Recueil de synonymes, un Traité des figures de langage, et autres ouvrages semblables. [...]

Quoi qu'il en soit, ces Études et ce Commentaire sur Racine, car c'est tout à la fois l'un et l'autre, ont particulièrement pour objet la diction et le style; et par diction, il faut le dire enfin, j'entends ici l'emploi et la disposition des mots conformément au génie de la langue et aux lois de l'usage ; par style, j'entends l'art de faire servir les mots et toutes les ressources de la langue à exprimer la pensée et le sentiment de la manière la plus vraie et la plus heureuse. Ce n'est donc pas un travail purement grammatical, comme les Remarques d'olivet; mais un travail tout à la fois grammatical et littéraire, où les lois du goût ne sont pas moins consultées que celles de la Syntaxe. Les défauts de Racine y tiennent plus de place que ses beautés, et la raison en est simple : les beautés sont bien moins faites pour être analysées que pour être senties, et, pour être senties, elles n'ont le plus souvent qu'à paraître. [...] Les constructions irrégulières, louches, embarrassées ; les tours de phrase peu naturels ; les termes impropres ou hors d'usage ; les ellipses forcées ou trop hardies; les métaphores et les personnifications peu justes ou peu cohérentes, enfin les incorrections, les négligences qui peuvent blesser un goût délicat : tels sont en général les défauts que je me suis occupé de relever; mais en me renfermant dans le même cercle que les divers Commentateurs, c'est-à-dire, en ne 
regardant jamais qu'à ce qui a été signalé par les uns ou par les autres, et en m'interdisant rigoureusement de rien examiner de ce qui a échappé à leur critique ou à leurs éloges. [...]

7 Du reste, ce n'est point indirectement ni en Historien que je rapporte les opinions des divers Commentateurs; mais je fais presque toujours parler les Commentateurs euxmêmes, et je les fais parler d'après le propre texte de leurs écrits. Sont-ils opposés entre eux sur quelque point, je les mets aux prises les uns avec les autres, et puis j'interviens souvent moi-même pour donner gain de cause à celui-ci ou à celui-là, ou même pour les combattre tous, suivant que la raison et la vérité me semblent l'exiger. Cette forme de l'ouvrage m'a paru plus piquante que toute autre que j'aurais pu employer, et j'ai dû d'autant moins balancer à la préférer qu'elle donne nécessairement lieu à une sorte d'intérêt dramatique. [...] Enfin, on ne reste pas simple lecteur, simple spectateur, mais on entre dans la lice, on prend part aux débats, et, suivant l'occasion, on attaque, on défend, on soutient, ou est pour, on est contre, on devient partie, juge, ou arbitre. [...]

Ces sortes de travaux ne me conduiront pas à l'immortalité, je le sais, et les grands noms auxquels j'attache le mien, ne le sauveront ni de l'obscurité, ni de l'oubli. Mais quel est mon but? quelle est mon ambition ? C'est uniquement de pouvoir être un peu utile en fournissant quelques secours pour de bonnes études en Grammaire et en Littérature. Si ce but n'est point manqué, ni cette ambition déçue, je suis assez content. Passionné dans ma jeunesse pour la gloire littéraire, j'eusse probablement, dans des circonstances plus favorables à mes premiers goûts, aspiré aux palmes de la poésie ou à celles de l'éloquence. Mais je suis aujourd'hui plus que consolé de n'avoir point paru dans la carrière, dès que, sans doute, je ne m'y fusse point distingué de la foule. N'y a-til pas assez de poètes ou d'orateurs médiocres, sans moi ? [FIN]

\section{Commentaire : articles 10-17, Phèdre (acte I, scène III)}

10.

Que ces vains ornements, que ces voiles me pèsent !

L.H. [Laharpe] Des voiles qui pèsent ! Quelle vérité d'idée dans cette espèce de contre-vérité d'expression! Cette singulière espèce de beauté n'est qu'indiquée dans le grec, qui dit seulement: Je souffre avec peine le voile qui couvre ma tête; mais Denys d'Halicarnasse remarque une intention imitative dans le commencement du vers grec, comme il y en a une dans les dernières syllabes $d u$ vers français. Le vers grec commence par une sorte de pied composé de deux brèves et d'une longue (l'anapeste), en sorte que le vers semble tomber à la troisième syllabe, comme la tête de Phèdre. Voilà de ces finesses de diction et d'harmonie qui doivent souvent échapper aux modernes dans les écrits des Anciens.

Le fond de cette remarque a été fourni par Louis Racine, et M. de Laharpe n'en dit rien, lui qui ne pardonnerait pas à Luneau ces sortes de réticences. Il y a toutefois cette contre-vérité d'expression dont Louis Racine ne s'était point avisé. Mais cette contre-vérité est-elle bien réelle? Y a-t-il véritablement une sorte d'opposition de mots dans ces ornements et dans ces voiles qui pèsent? Il me semble que me pèsent peut signifier aussi bien, et même plutôt, m'incommodent, m'embarrassent, m'importunent, que pèsent sur moi, me surchargent, m'accablent. Cela n'empêche pas que le vers ne soit toujours très beau, et pour l'expression, et pour l'harmonie. Qu'il me soit permis de rappeler, en passant, 
l'heureuse application qu'en fait Marmontel, relativement à l'usage des épithètes (Encyclopédie méthodique, article Épithète du Supplément). "Quand la passion vient se saisir de toutes les facultés de l'âme, et l'occuper de son objet unique, tout ce qui n'ajoute pas à l'intérêt de l'expression lui est étranger. Elle rebute les mots de pure ostentation, elle dédaigne le soin de plaire. Son unique soulagement est de se répandre au-dehors. L'épithète qui l'aide à s'exprimer lui devient précieuse ; celle qui ne ferait que la distraire, la ralentir, la refroidir, la gênerait; et, comme Phèdre, la nature dirait alors : Que ces vains ornements, que ces voiles me pèsent !»

11.

Quelle importune main, en formant tous ces nœuds,

A pris soin sur mon front d'assembler mes cheveux?

L. Rac. [Louis Racine] De les arranger avec art. Cependant arranger serait ici un mot faible: assembler présente une image. Elle (Phèdre) devrait être les cheveux épars ; c'est une main étrangère et importune qui les a assemblés.

Que les nœuds soient de cheveux ou de tout autre chose, on n'a pu les former qu'après avoir assemblé les cheveux. Le poète eût donc pu faire dire à Phèdre, avec autant de vérité et de justesse :

Quelle importune main, assemblant mes cheveux,

A pris soin sur mon front de former tous ces nœuds?

11 Mais alors l'action d'assembler les cheveux ne devenait qu'une circonstance de la phrase, et il valait beaucoup mieux la présenter comme l'objet principal : il le fallait même pour faire entendre, non seulement que ces cheveux, maintenant assemblés sur le front, devraient être épars, mais qu'ils l'étaient naguère en signe de douleur et de deuil.

12.

Dieux ! que ne suis-je assise à l'ombre des forêts !

Quand pourrai-je, au travers d'une noble poussière,

Suivre de l'œil un char fuyant dans la carrière ?...

L.B. [Luneau de Boisjermain] Ceci est une traduction vive et rapide d'Euripide. "Hélas! dit Phèdre, que ne puis-je à présent m'égarer dans les bois, etc. etc. »

L.H. On voit qu'Euripide est toujours trop long, et Racine toujours précis.

L.R. [Louis Racine] On est obligé de prononcer rapidement ce vers, suivre de l'œil, etc.

Ce qui était bien plus utile à remarquer, peut-être, c'est ce détour adroit par lequel Phèdre, pressée de s'ouvrir à Enone, la met sur la voie de deviner ce qu'elle n'oserait lui déclarer ouvertement, c'est-à-dire, sa passion criminelle pour Hippolyte, qui faisait sa principale occupation de la chasse, et excellait à conduire un char rapide. Un fameux écrivain du dernier siècle, connu surtout par la grande part qu'il a eue à l'Encyclopédie, en était si émerveillé qu'il a dit : "Le poète n'a pu se promettre ce morceau qu'après l'avoir trouvé, et je m'estime plus d'en sentir le mérite, que de quelque chose que je puisse écrire de ma vie.» 
13.

Les ombres par trois fois ont obscurci les cieux, Depuis que le sommeil n'est entré dans vos yeux, Et le jour a trois fois chassé la nuit obscure,

Depuis que votre corps languit sans nourriture.

L.H. Ces quatre vers peuvent paraître pompeux, et c'est l'avis de Luneau. Observez cependant que, s'il est plus court de dire, il y a trois jours que vous n'avez mangé ni dormi, d'un autre côté le dire en quatre vers, qui ne sont que poétiques sans être pompeux, a pour objet de rendre, par la longueur de la phrase, la longueur de l'abstinence et de l'insomnie. Il n'y a donc que de la vérité, et point d'affectation. C'est ce qu'il était bon de noter contre ceux qui s'autoriseraient de ces quatre vers pour faire de la poésie mal à propos.

Je veux que ces quatre vers ne soient que poétiques : mais ne le sont-ils pas trop pour la circonstance? Ne le sont-ils pas trop dans la bouche d'une suivante, et d'une suivante affligée de la douleur de sa maîtresse? Je veux que ces deux périphrases peignent mieux la longueur de l'abstinence et de l'insomnie, que ne le ferait l'expression simple, depuis trois jours et depuis trois nuits, vous n'avez ni mangé ni dormi : mais cette symétrie et cette sorte d'antithèse du premier vers de la seconde périphrase avec le premier vers de la première! N'est-il rien là que de bien vrai, que de bien naturel, que de bien conforme au langage de la douleur? Je ne sais, mais il me semble que ce serait déplacé, même dans un récit purement épique. J'aimerais mieux, je l'avoue, que le poète n'eût énoncé le temps qu'une seule fois, et que, réduisant les autre vers à trois, il eût dit, par exemple :

Les ombres par trois fois ont obscurci les cieux,

Depuis que le sommeil n'est entré dans vos yeux,

Depuis que votre corps languit sans nourriture ;

ou bien :

Le jour a trois fois chassé la nuit obscure

Depuis que votre corps languit sans nourriture,

Depuis que le sommeil n'est entré dans vos yeux.

Qu'on rapproche des vers si poétiques d'Ænone, les vers si simples où Josabeth, dans Athalie, Acte $1^{\text {er }}$, Scène II, exprime que, depuis trois jours et trois nuits, elle ne fait que jeûner et prier :

Surtout j'ai cru devoir aux larmes, aux prières,

Consacrer ces trois jours et ces trois nuits entières.

14.

A quel affreux dessein vous laissez-vous tenter?

L.B. On ne dit point se laisser tenter à quelque chose.

L.H. Je suis convaincu qu'on le dit très souvent et très bien. Se laisser tenter à... , pour se laisser aller à la tentation de..., est une phrase proprement française, une espèce de gallicisme elliptique. Je ne voulais pas aller à la comédie, mais je me suis laissé tenter à Phèdre. 
G.F. [Geoffroy] Se laisser tenter à quelque chose est contraire à la Grammaire et n'a rien de favorable à la poésie. M. de Laharpe a essayé de justifier cette façon de parler. Il a pris la peine de faire lui-même une phrase pour servir d'exemple et d'autorité... Cette phrase, aussi ridicule que malheureuse, est sa propre condamnation. condamne-t-elle Laharpe ? En quoi est-elle si malheureuse et si ridicule! Se laisser tenter à quelque chose contraire à la Grammaire! Et depuis quand? Une chose ne peut-elle pas nous tenter, comme elle peut nous séduire, nous entraîner, nous emporter, nous vaincre ? Or, ne dit-on pas très bien (j'en appelle au Dictionnaire de l'Académie), se laisser séduire à une chose, s'y laisser entraîner, s'y laisser emporter, s'y laisser vaincre ? Voir dans Iphigénie ce qui a été dit sur ce vers :

Je me laisse conduire à cet aimable guide.

15.

Grâces au ciel, mes mains ne sont point criminelles.

Plût aux Dieux que mon cœur fût innocent comme elles !...

L. H. Le pronom elles ne doit point d'ordinaire se trouver à la fin d'une phrase quand il se rapporte aux choses, mais seulement quand il se rapporte aux personnes : le contraire n'est pas un solécisme, mais une faute d'élégance. Ici ces mots comme elles sont si bien placés et vont si bien au sens, qu'il n'y a rien à dire. Mais je ne sais si l'on rencontrerait dans Racine, depuis Andromaque, un autre endroit où il ait manqué à cette règle de diction, dont Voltaire parait avoir tenu bien peu de compte dans sa versification. Vous lisez dans Tancrède :

Mais qui peut altérer vos bontés paternelles?

Vous seule, vous, ma fille, en abusant trop d'elles.

Qui est-ce qui ne sent combien cet elles, placé là, rend la phrase lâche et complaisante? Cette construction ne serait pas supportable, même en prose. écrite, et $\mathrm{M}$. de Laharpe n'ignorait pas que celles dont ce grand poète a un peu soigné la versification, n'offrent pas ce défaut, qu'on ne trouverait pas une seule fois, je crois, dans toute la Henriade.

te, pourquoi cet elles est-il si mal placé dans les deux vers de Tancrède? Parce que les bontés paternelles auxquelles il se rapporte, ne sont présentées que comme une simple abstraction, et qu'il n'y a rien, ni dans le premier vers, ni dans le second, qui tende même à les personnifier. Pourquoi le même pronom est-il, au contraire, très bien à sa place dans les vers de Racine? Parce que les mains, auxquelles il se rapporte, sont personnifiées jusqu'à un certain point, comme le cœur, par cette espèce de sentiment et d'intention que semblent leur prêter les mots criminelles et innocent. Et cette personnification, soit du cœur, soit de la main même, est très ordinaire, non seulement en poésie, mais même dans la prose élevée, parce que le cœur concevant le crime, et la main l'exécutant, il est assez naturel de les regarder comme y prenant une part active.

16.

Songez-vous qu'en naissant mes bras vous ont reçue? 
L.H. Le gérondif en naissant se rapporte par le sens à Phèdre, et par la construction à Enone. C'est une faute de Grammaire, excusable en faveur de la clarté et de la précision du vers, mais qu'il ne faudrait se permettre qu'avec la plus grande réserve, et avec les mêmes excuses bien avouées. Racine se l'est très rarement permise.

Pas tout à fait si rarement, et plus souvent, peut-être, que d'autres classiques dont le style n'a pas, à beaucoup près, la même réputation de pureté et d'exactitude. Voir dans Britannicus ce qui a été dit sur les vers :

Mes soins en apparence épargnant ses douleurs,

De son fils, en mourant, lui cachèrent les pleurs.

17.

Un reste de chaleur tout prêt à s'exhaler.

L.H. Dans tout ce morceau sublime de passion et de style, depuis ces mots, mon mal vient de plus loin, etc., rien n'est emprunté d'Euripide ; mais le poète, toujours plein de l'esprit des Anciens, a fondu dans ce couplet quelques-uns des vers les plus passionnés que l'antiquité nous ait laissés. Celui de Virgile :

Ut vidi! ut perii ! ut me malus abstulit error!

Je le vis, je rougis, je pâlis à sa vue.

Celui d'Horace :

In me tota ruens Venus.

C'est Vénus toute entière à sa proie attachée.

Et trois vers de la fameuse Ode de Sapho, traduite par Boileau [dans le Traité $\mathrm{du}$ sublime de Longin, $\mathrm{X}, 2$, Je sens de veine en veine une subtile flamme / Courir par tout mon corps sitôt que je te vois], mais qui sont rendus ici avec plus de noblesse et d'élégance.

Un trouble s'éleva dans mon âme éperdue.

Mes yeux ne voyaient plus, je ne pouvais parler ;

Je sentis tout mon corps et transir et brûler.

Et dans tous ces endroits imités, Racine me paraît supérieur aux originaux ; et quels originaux! Et dans ce qui est à lui, il n'est pas au-dessous. On convient généralement que la scène entière est un modèle étonnant de toutes les beautés tragiques et poétiques dans leur perfection: intérêt, dialogue et style, tout $y$ est au plus haut point. Une pareille scène pèse plus d'une tragédie, et Racine en a plus d'une de ce poids.

Louis Racine, qui a fourni le fond de cet article, rapporte, avant le vers si énergique,

[...], C'est Vénus toute entière à sa proie attachée.

celui dont il est précédé,

Ce n'est plus une ardeur dans mes veines cachée :

[...].

et il y joint une observation qui me paraît pouvoir contribuer à faire connaître le génie de notre langue. « La Phèdre de Garnier disait :

L'amour consomme enclos

L'humeur de ma poitrine, et dessèche mes os ;

Il rage en ma moëlle, et le cruel m'enflamme

Le cœur et les poumons d'une mortelle flamme. 
Notre style noble ne reçoit plus moëlle, poumons, poitrine. Vaugelas prétend que poitrine est proscrit, parce qu'on dit une poitrine de veau. Plusieurs autres mots sont proscrits du style noble, sans qu'on en puisse donner de raisons, comme sueur, moëlle, les poumons, les entrailles, si ce n'est au figuré. L'estomac n'a pas plus de privilège, quoique Corneille l'ait fait entrer dans un vers de Rodogune:

D'une profonde plaie, en l'estomac ouverte.

Nous n'osons nommer les nerfs, et nous nommons élégamment les veines :

Ce n'est plus une ardeur dans mes veines cachée...

Juste ciel ! tout mon sang dans mes veines se glace ! Esther.

Je sens de veine en veine une subtile flamme [...]. Boileau "

Voilà ce que dit Louis Racine, et c'est en général assez juste, avec cette restriction, toutefois, que l'art et le talent parviennent souvent à donner de la noblesse à ce qui en paraissait le moins susceptible. Est-ce que sueur, par exemple, n'est pas très heureusement placé dans ces vers de Delille, traduction des Géorgiques, livre III :

Le coursier, l'œil éteint et l'oreille baissée,

Distillant lentement une sueur glacée,

Languit, chancelle, tombe, et se débat en vain.

\section{AUTEUR}

\section{PIERRE FONTANIER}

$1765-1844$ 